\title{
Entanglement assisted capacity of the broadband lossy channel
}

\author{
Vittorio Giovannetti ${ }^{1}$, Seth Lloyd ${ }^{1,2}$, Lorenzo Maccone ${ }^{1}$, and Peter W. Shor ${ }^{3}$ \\ Massachusetts Institute of Technology $-{ }^{1}$ Research Laboratory of Electronics, ${ }^{2}$ Department of Mechanical Engineering \\ 77 Massachusetts Ave., Cambridge, MA 02139, USA \\ ${ }^{3}$ ATET Labs - Research 180 Park Ave, Florham Park, NJ 07932, USA
}

(Dated: February 9, 2020)

\begin{abstract}
We calculate the entanglement assisted capacity of a multimode bosonic channel with loss. As long as the efficiency of the channel is above $50 \%$, the superdense coding effect can be used to transmit more bits than those that can be stored in the message sent down the channel. Bounds for the other capacities of the multimode channel are also provided.

PACS numbers: 03.67.Hk,42.50.-p,89.70.+c,05.40.Ca
\end{abstract}

Among the zoology of different capacities of quantum channels [1, 2], the entanglement assisted classical capacity $C_{E}$ plays an important role. This quantity has been introduced in [3] to measure the amount of classical information that can be sent through the channel in the presence of an unlimited quantity of prior entanglement between sender and receiver. $C_{E}$ and its quantum counterpart $Q_{E}=C_{E} / 2$ (i.e. the amount of qubits that can be sent in the presence of an unlimited quantity of prior entanglement) give upper bounds to the classical and quantum capacities of the channel, including the unassisted capacities whose values are yet to be determined. Moreover, it has been conjectured [4] that the entanglement assisted classical capacity defines a class of equivalences since all channels with the same $C_{E}$ seem to be able to efficiently simulate one another. Unlike the case of most of the other capacities, it has a closed expression in terms of the quantum mutual information

$$
I(\mathcal{N}, \varrho)=S(\varrho)+S(\mathcal{N}[\varrho])-S\left((\mathcal{N} \otimes \mathbb{1})\left[\Phi_{\varrho}\right]\right),
$$

where $S(\varrho)=-\operatorname{Tr}\left[\varrho \log _{2} \varrho\right]$ is the Von Neumann entropy, $\mathcal{N}$ is the map that describes the communication channel and $\Phi_{\varrho}$ is a purification of the input density matrix $\varrho$. The value of $C_{E}$ is the maximum of $I(\mathcal{N}, \varrho)$ over all the possible inputs $\varrho$ to the channel [4, [5].

The entanglement assisted capacity for bosonic Gaussian channels was analyzed in [6], where it was shown that the maximization in the expression of $C_{E}$ can be performed over Gaussian states. These channels are important because they are the basic building blocks of bosonic communication schemes and because they allow one to describe infinite dimensional systems with techniques from finite dimensional linear algebra. In this paper we derive $C_{E}$ for multimode bosonic channels in the presence of loss and average input energy constraint, and use these results and the techniques developed to provide upper and lower bounds for other channel capacities. We calculate $C_{E}$ for the multimode channel as the sum of the entanglement assisted capacities of the single modes maximized over non-squeezed Gaussian states. In fact, $C_{E}$ is additive and we show that squeezing the input states does not increase the $C_{E}$ of a single mode. For generic values of the channel quantum efficiency $\eta$ we cannot provide an analytical expression for $C_{E}$, but we give a general characterization and a numerical solution. For $\eta=1 / 2$, the value of $C_{E}$ can be analytically solved and, interestingly, shown to coincide with the wideband lossless channel capacity [7].

Broadband lossy channel. - In the Heisenberg picture the $i$ th mode of the lossy channel with quantum efficiency $\eta_{i}$ evolves as

$$
a_{i}^{\prime}=\sqrt{\eta_{i}} a_{i}+\sqrt{1-\eta_{i}} b_{i}
$$

where $a_{i}, a_{i}^{\prime}$ and $b_{i}$ are the annihilation operators of the input, output and noise modes respectively. The loss map $\mathcal{N}_{i}$ for the $i$ th mode arises by tracing away the noise mode $b_{i}$ (in the vacuum state) and the global loss map $\mathcal{N}$ is the tensor product $\bigotimes_{i} \mathcal{N}_{i}$. The channel described by $\mathcal{N}$ maps Gaussian input states into Gaussian output states and is hence a Gaussian channel.

The calculation of $C_{E}$ for the multimode lossy channel stems from the following three facts: i) the additivity property of the entanglement assisted capacity, from which the $C_{E}$ of the channel is calculated as the sum of the $C_{E}$ of each mode [1, 8], i.e.

$$
C_{E}=\max _{\varrho_{j} \in \mathcal{H}_{j}}\left\{\sum_{i} I\left(\mathcal{N}_{i}, \varrho_{i}\right)\right\}
$$

where $\mathcal{H}_{j}$ is the Hilbert space of the $j$ th mode of the channel, and the max is taken over the states $\varrho_{i}$ that satisfy the average energy constraint

$$
\sum_{i} \hbar \omega_{i} N_{i}=\mathcal{E}
$$

with $\omega_{i}$ the frequency of the $i$ th mode and $N_{i}$ its average number of photons; ii) the Holevo-Werner theorem according to which the maximum of $I\left(\mathcal{N}_{i}, \varrho_{i}\right)$ for Gaussian channels can be evaluated on Gaussian input states [6]; iii) the fact that squeezing the input does not increase $C_{E}$, so that it can be estimated on non-squeezed inputs: as shown in the appendix, the maximum value of $I\left(\mathcal{N}_{i}, \varrho_{i}\right)$ (fixing the energy in the $i$ th mode) is obtained when $\varrho_{i}$ does not contain any squeezing and is given by

$$
c_{E}\left(N_{i}, \eta_{i}\right)=g\left(N_{i}\right)+g\left(\eta_{i} N_{i}\right)-g\left(\left(1-\eta_{i}\right) N_{i}\right),
$$


where the function $g$ is defined as

$$
g(x) \equiv(x+1) \log _{2}(x+1)-x \log _{2}(x),
$$

for $x \neq 0$ and $g(0)=0$. The total entanglement assisted capacity is then

$$
C_{E}=\max _{N_{j}} \sum_{i} c_{E}\left(N_{i}, \eta_{i}\right),
$$

where the maximum is taken over the sets $\left\{N_{j}\right\}$ satisfying the energy constraint (44).

The maximization (7) can be performed using the Lagrange multiplier procedure, which, for $\eta \neq 0,1$, gives the following equation [9]

$$
\begin{aligned}
\left(1+\frac{1}{N_{j}}\right) & \left(1+\frac{1}{\eta_{j} N_{j}}\right)^{\eta_{j}} \\
= & e^{\omega_{j} / \Omega}\left(1+\frac{1}{\left(1-\eta_{j}\right) N_{j}}\right)^{1-\eta_{j}},
\end{aligned}
$$

where $1 /(\Omega \ln 2)$ is the Lagrange multiplier that must be chosen to satisfy the constraint (4). In general this equation is difficult to solve analytically, but we can still give some characterization of the solution, at least when all the quantum efficiencies coincide (i.e. $\eta_{j}=\eta$ for all $j$ ). In this case the solution of Eq. (8) is a function of $\omega_{j} / \Omega$ and $\eta$, i.e. $N_{j}=\mathcal{F}\left(\omega_{j} / \Omega, \eta\right)$. To derive $\Omega$ we use Eq. (4) that becomes

$$
\frac{\mathcal{E}}{\hbar}=\sum_{i} \omega_{i} \mathcal{F}\left(\omega_{i} / \Omega, \eta\right) \simeq \int_{0}^{\infty} \frac{d \omega}{\delta \omega} \omega \mathcal{F}(\omega / \Omega, \eta),
$$

where we have replaced the sum over the mode index $i$ with an integral over the mode frequencies, assuming that the minimum frequency interval $\delta \omega$ of the channel is small. With a variable change in the integral (9), we find that $\Omega=\sqrt{2 \pi \mathcal{P} /[f(\eta) \hbar]}$ where $\mathcal{P}=\mathcal{E} \delta \omega /(2 \pi)$ is the wideband channel input power during the transmission time $\mathcal{T}=2 \pi / \delta \omega$ and

$$
f(\eta) \equiv \int_{0}^{\infty} d x x \mathcal{F}(x, \eta) .
$$

The value of $C_{E}$ is then obtained placing the solution of Eq. (8) to evaluate the sum (7), i.e.

$$
C_{E} \simeq \int_{0}^{\infty} \frac{d \omega}{\delta \omega} c_{E}(\mathcal{F}(\omega / \Omega, \eta), \eta) .
$$

Performing again a change of integration variables, we finally find

$$
C_{E}=\mathcal{T} \frac{1}{\ln 2} \sqrt{\frac{\pi \mathcal{P}}{3 \hbar}} \mathcal{C}(\eta)
$$

where

$$
\mathcal{C}(\eta) \equiv \frac{\ln 2}{\pi} \sqrt{\frac{3}{2 f(\eta)}} \int_{0}^{\infty} d x c_{E}(\mathcal{F}(x, \eta), \eta) .
$$

Notice that, even without knowing the explicit form of the function $\mathcal{C}(\eta)$, Eq. (12) gives the exact dependence on the input power of the entanglement assisted capacity for the channel 10]. In particular, the entanglement assisted capacity per unit time of channel use $R_{E} \equiv C_{E} / \mathcal{T}$ is proportional to the rate $R_{C}=\frac{1}{\ln 2} \sqrt{\frac{\pi \mathcal{P}}{3 \hbar}}$ of the wideband noiseless bosonic channel without prior entanglement [7], i.e. $R_{E}=R_{C} \mathcal{C}(\eta)$.

General properties of $C_{E}$. - The form of $\mathcal{C}(\eta)$ is not easily determined analytically, but we can still calculate it for some values of $\eta$. First of all, for $\eta=0$ all the $c_{E}\left(N_{i}, \eta\right)$ are null and $\mathcal{C}(0)=0$ : no photons arrive, and no bits are transferred. Interestingly, for $\eta=1 / 2$ Eq. (8) can be solved analytically and has solution

$$
N_{j}=\frac{1}{e^{\omega_{j} / \Omega}-1} .
$$

In this case, $f(1 / 2)=\pi^{2} / 6$ and $\mathcal{C}(1 / 2)=1$, and hence the entanglement assisted capacity for the $\eta=1 / 2$ wideband channel equals the unassisted capacity of the noiseless wideband channel $\mathcal{T} R_{C}$ [7]: prior entanglement is sufficient to restore perfect transmission for a $50 \%$ lossy channel (this result holds also for the single mode channel- see appendix). The solution can be linearized around $\eta=1 / 2$ and the first order Taylor expansion of $\mathcal{C}(\eta)$ can be obtained as

$$
\mathcal{C}(\eta)=\frac{3}{2}\left(\eta-\frac{1}{2}\right)+1+\mathcal{O}\left((\eta-1 / 2)^{2}\right) .
$$

The case $\eta=1$ can be completely solved too, given that the Lagrange equation has the same solution (14) of the case $\eta=1 / 2$. Here, since $c_{E}\left(N_{i}, 1\right)=2 c_{E}\left(N_{i}, 1 / 2\right)$, we find $\mathcal{C}(1)=2 \mathcal{C}(1 / 2)=2$ : the entanglement assisted capacity for the noiseless channel is twice the unassisted capacity as predicted by the superdense coding effect 11]. In Fig. 1h $\mathcal{C}(\eta)$ is numerically evaluated and plotted along with the linearization (15). The fact that $\mathcal{C}(\eta)>1$ for $\eta>1 / 2$ shows that, even in the presence of noise, prior entanglement allows one to transmit more bits than those actually sent in the channel (i.e. $\mathcal{T} R_{C}$ ) thanks again to the superdense coding effect. A similar effect has been shown also for the erasure channel 3,12 .

An interesting class of lower bounds, that provides a good analytical approximation for $C_{E}$ can be obtained by considering the set (parametrized by $\zeta>0$ )

$$
N_{j}=\frac{\zeta^{2}}{e^{\zeta \omega_{j} / \Omega_{0}}-1},
$$

where $\Omega_{0}=6 \ln 2 R_{C} / \pi$. Using Eq. (16), we find the bound

$$
\mathcal{C}(\eta) \geqslant\left[\Lambda\left(\zeta^{2}\right)+\Lambda\left(\eta \zeta^{2}\right)-\Lambda\left((1-\eta) \zeta^{2}\right)\right] /[\zeta \Lambda(1)],
$$

where $\Lambda(y) \equiv \int_{0}^{\infty} d x g\left(\frac{y}{e^{x}-1}\right)$. In particular, the case $\zeta=1$ (see Fig. [1 $)$ ) corresponds to employing the exact solution for $\eta=1 / 2,1$ of Eq. (14) for any value of $\eta$. 


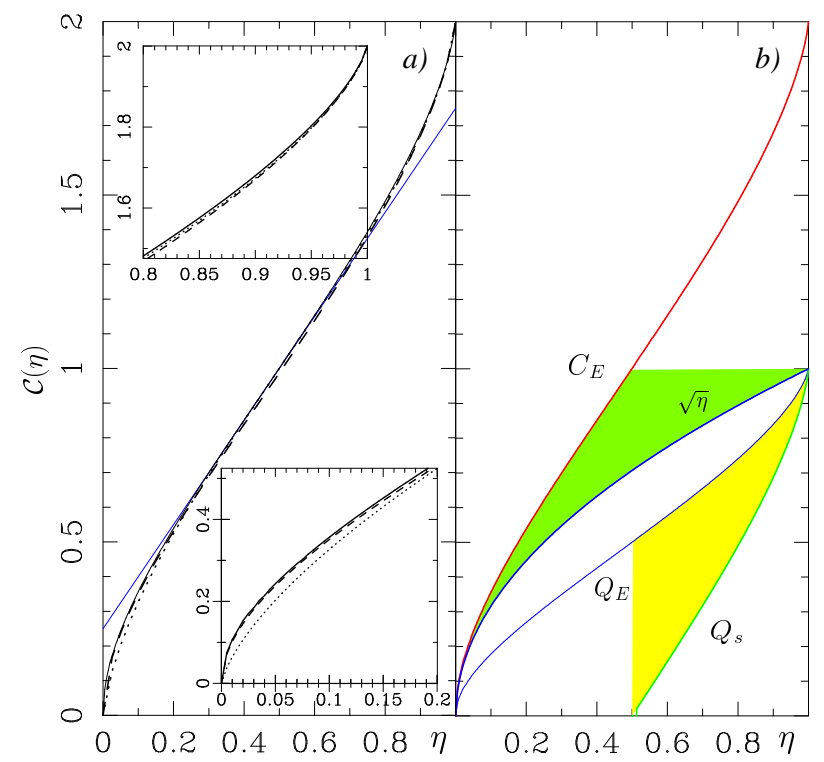

FIG. 1: a) Plot, as function of the quantum efficiency $\eta$, of the numerical solution for $\mathcal{C}(\eta)$ of Eq. (13) (continuous line), of the linearization (15) (gray line), and of the lower bounds (17) with $\zeta=1$ (dotted line) and $\zeta=1 / \sqrt{\eta}$ (dashed line). The inserts show the same graph in the regions of small and large $\eta$. The points above $\mathcal{C}(\eta)=1$ (i.e. for $\eta>1 / 2$ ) show where the superdense coding effect allows a lossy channel to beat the capacity of the noiseless channel without prior entanglement. b) Classical and quantum capacities of the lossy wideband channel. The classical capacity $C /\left(\mathcal{T} R_{C}\right)$ is confined in the dark gray area between the upper bound given by $C_{E}$ and the lower bound $\sqrt{\eta}$. The quantum capacity $Q /\left(\mathcal{T} R_{C}\right)$ is confined in the light gray area between the upper bound given by the entanglement assisted quantum capacity $Q_{E}=C_{E} / 2$ and the lower bound $Q_{s}$ obtained by calculating the coherent information according to Eq. (19). $Q$ is null for $\eta \leqslant 1 / 2$.

Capacity bounds. - The classical capacity $C$ and the quantum capacity $Q$ measure respectively the number of bits and qubits that can be sent reliably through the channel per channel use (without the aid of prior entanglement). Unlike the case of $C_{E}$, for $\eta \neq 1$ a closed expression for $C$ is not known nor it is known whether this quantity is additive [1]: it may be that entangling successive uses of the channel one can increase the amount of information transmitted. Limiting the analysis to unentangled coding procedures, a lower bound for $C$ can be obtained as 13]

$$
C \geqslant \max _{p_{j}(\mu), \rho_{j}(\mu)} \sum_{i} \mathcal{X}\left(p_{i}(\mu), \rho_{i}(\mu)\right),
$$

where $\varrho_{i}=\int d \mu p_{i}(\mu) \rho_{i}(\mu)$ describes a message in which the " $\mu$ th letter" $\rho_{i}(\mu)$ in the $i$ th mode has probability density $p_{i}(\mu)$ and where $\mathcal{X}$ is the Holevo information $S\left(\mathcal{N}_{i}\left[\varrho_{i}\right]\right)-\int d \mu p_{i}(\mu) S\left(\mathcal{N}_{i}\left[\rho_{i}(\mu)\right]\right)$. To estimate the lower bound in Eq. (18), we follow the suggestion of [6] and we evaluate $\mathcal{X}\left(p_{i}(\mu), \rho_{i}(\mu)\right)$ for the $i$ th mode using coherent states $\rho_{i}(\mu)=|\mu\rangle_{i}\langle\mu|$ weighted with Gaussian probabil- ity distribution $p_{i}(\mu)=\exp \left[-|\mu|^{2} / N_{i}\right] /\left(\pi N_{i}\right), N_{i}$ being the average number of photons of the mode. In this case, Eq. (18) becomes $C \geqslant \max _{N_{j}} \sum_{i} g\left(\eta_{i} N_{i}\right)$, where again the maximum must be taken under the average energy constraint (44). The corresponding Lagrange equation has solution given by Eq. (16) with $\zeta=1 / \sqrt{\eta}$, so that $C \geqslant \mathcal{T} \sqrt{\eta} R_{C}$ [14] (see Fig. 10). Notice that for $\eta=1$ the equality holds, since the noiseless channel is known to be additive and we reobtain the results of [7]. A closed expression for $Q$ is also not known. However, for $\eta \leqslant 1 / 2$ the no-cloning theorem can be used to show that $Q=0$, as in the case of the erasure channel [12, 14]. For $\eta>1 / 2$, a lower bound can be obtained evaluating the coherent information $J(\mathcal{N}, \varrho)=S(\mathcal{N}[\varrho])-S\left((\mathcal{N} \otimes \mathbb{1})\left[\Phi_{o}\right]\right)$ on unentangled non-squeezed Gaussian inputs 14, 15. In fact, random quantum codes can send quantum information down a noisy channel at a rate given by the coherent information [16]. In Fig. [1 this bound is plotted by solving numerically the corresponding Lagrange equation, which maximizes the expression

$$
Q \geqslant \max _{N_{j}} \sum_{i} g\left(\eta N_{i}\right)-g\left((1-\eta) N_{i}\right) .
$$

Conclusions. - Up to now only few realistic channels have been analyzed at the quantum level. In this paper we studied the wideband bosonic channel with loss, calculating the entanglement assisted capacities $C_{E}$ and $Q_{E}$ and we gave upper and lower bounds on the classical and quantum capacities of this channel. The capacity $C_{E}$ was shown to scale with the square root of the input power as shown previously for the classical capacities in the noiseless case. Moreover, we saw that the superdense coding effect allows the sender to increase the information transferred above the entropy of the input state if the quantum efficiency is $\eta>1 / 2$.

This work was funded by the ARDA, NRO, NSF, and by ARO under a MURI program.

Appendix. - In [6] it has been shown that, for a given value of the correlation matrix $\alpha$, the quantum mutual information $I(\mathcal{N}, \varrho)$ for a single mode $a$ achieves its maximum value on the Gaussian state

$$
\varrho=\frac{\hbar}{2 \pi} \int d z \exp \left[-i(\Delta q, \Delta p) \cdot z^{T}-z \cdot \alpha \cdot z^{T} / 2\right],
$$

where $z$ is a real bidimensional line vector and $q$ and $p$ the two orthogonal quadratures $q=\sqrt{\hbar / 2}\left(a+a^{\dagger}\right)$, $p=-i \sqrt{\hbar / 2}\left(a-a^{\dagger}\right)$. In order to evaluate the effect of the squeezing on the quantum mutual information of the single mode channel, it is convenient to introduce the following parametrization for the correlation matrix $\alpha$ :

$$
\alpha=\frac{\hbar}{2}\left[\begin{array}{cc}
n_{0} e^{r} & c \\
c & n_{0} e^{-r}
\end{array}\right],
$$

where $r$ is the squeezing parameter. These parameters are related through the average number of photons $N$ by 


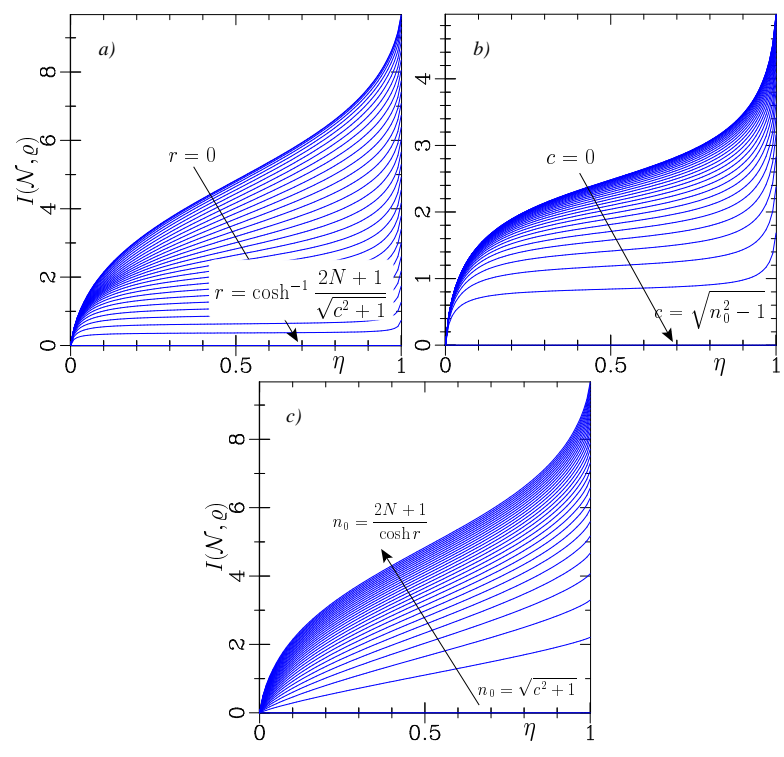

FIG. 2: Plots of the quantum mutual information $I(\mathcal{N}, \varrho)$ of Eq. (22): a) $I(\mathcal{N}, \varrho)$ decreases with $r$ (here $c=0, m=0$ ); b) $I(\mathcal{N}, \varrho)$ decreases with $c$ (here $r=0, m=0)$; c) $I(\mathcal{N}, \varrho)$ increases with $n_{0}$, i.e. decreases with $m$ (here $r=0, c=0$ ). In all plots $N=10$.

the conditions $\sqrt{c^{2}+1} \leqslant n_{0}=[(2 N+1)-m] / \cosh r$ : the first relation derives from the strong version of the uncertainty relation, while the second from the average energy constraint (with $m=\langle q / \hbar\rangle^{2}+\langle p / \hbar\rangle^{2}$ ). With these definitions the quantum mutual information becomes

$$
\begin{aligned}
& I(\mathcal{N}, \varrho)=g(\gamma(1))+g(\gamma(\eta))-g(\gamma(1-\eta)) \\
& \gamma(\eta) \equiv \sqrt{\left[\eta \lambda_{+}+\frac{1-\eta}{2}\right]\left[\eta \lambda_{-}+\frac{1-\eta}{2}\right]}-\frac{1}{2}
\end{aligned}
$$

where $\lambda_{ \pm}=\frac{1}{2}\left[n_{0} \cosh (r) \pm \sqrt{\left(n_{0} \sinh (r)\right)^{2}+c^{2}}\right]$ are the two eigenvalues of $\alpha / \hbar$. Notice that for $\eta=1$, when all the photons reach the receiver, $I(\mathcal{N}, \varrho)$ is twice the entropy of the initial state, as predicted by the superdense coding effect [1]. In general, one can verify that $I(\mathcal{N}, \varrho)$ is smaller than the initial entropy for $\eta<1 / 2$ and greater for $\eta>1 / 2$ : the effect of superdense coding is, hence, evident only in this last case. Since the eigenvalues $\lambda_{ \pm}$are related with the average number of photons $N$ as

$$
\lambda_{+}+\lambda_{-}=2 N+1-m,
$$

one can show that the maximum of $I(\mathcal{N}, \varrho)$ for fixed $N$ is obtained for $\lambda_{+}=\lambda_{-}$. This is equivalent to requiring $r=0$ (i.e. no energy should be "wasted" in squeezing the input- see Fig. 2a) and $c=0$ (see Fig. 2b). This last condition attests that the best one can do to convey information is to send maximally mixed states, since the parameter $|c|$ measures the purity of the initial state. Choosing the maximum value of $c$ corresponds to sending a single pure state and conveys no information. Finally, since $I(\mathcal{N}, \varrho)$ is an increasing function of $n_{0}$, it can be further maximized by choosing $n_{0}=2 N+1$ (i.e. its maximum allowed value achieved when $\langle q\rangle=\langle p\rangle=0$ see Fig. 22). With this choice, Eq. (23) becomes $\gamma_{\text {opt }}(\eta)=$ $\eta N$, which maximizes the quantum mutual information as

$$
\begin{aligned}
c_{E}(\mathcal{N}, \varrho) & \equiv \max _{\varrho \mid\left\langle a^{\dagger} a\right\rangle=N} I(\mathcal{N}, \varrho) \\
& =g(N)+g(\eta N)-g((1-\eta) N),
\end{aligned}
$$

as reported in Eq. (5).

[1] C. H. Bennet and P. W. Shor, IEEE Trans. Inf. Theory 44, 2724 (1998).

[2] M. A. Nielsen and I. L. Chuang, Quantum Computation and Quantum Information (Cambridge University Press, Cambridge, 2000); J. Preskill, lecture notes at http://www.theory.caltech.edu/people/preskill/ph229

[3] C. H. Bennett, P. W. Shor, J. A. Smolin, and A. V. Thapliyal, Phys. Rev. Lett. 83, 3081 (1999).

[4] C. H. Bennett, P. W. Shor, J. A. Smolin, and A. V. Thapliyal, IEEE Trans. Inf. Theory 48, 2637 (2002), eprint quant-ph/0106052

[5] A. S. Holevo eprint quant-ph/0106075

[6] A. S. Holevo and R. F. Werner, Phys. Rev. A 63, 032312 (2001).

[7] H. P. Yuen and M. Ozawa, Phys. Rev. Lett. 70, 363 (1992); C. M. Caves and P. D. Drummond, Rev. of Mod. Phys. 66, 481 (1994).

[8] C. Adami, N. J. Cerf, Phys. Rev. A 56, 3470 (1997).

[9] For $\eta=0,1$ one has to apply the Lagrange procedure directly to the functions $c_{E}\left(N_{i}, 0\right)$ and $c_{E}\left(N_{i}, 1\right)$. This gives the same result one would get extending by continuity the solution of Eq. (8) under the constraint (4).

[10] Notice that, since we have replaced the summations with the integrals, Eq. (12) is valid up to corrections of order $1 / \mathcal{T}$

[11] C. H. Bennett and S. J. Wiesner, Phys. Rev. Lett. 69, 2881 (1992).

[12] C. H. Bennett, D. P. DiVincenzo, and J. A. Smolin, Phys. Rev. Lett. 78, 3217 (1997).

[13] P. Hausladen, R. Jozsa, B. Schumacher, M. Westmoreland, and W. K. Wootters, Phys. Rev. A 54, 1869 (1996); B. Schumacher and M. D. Westmoreland, Phys. Rev. A 56, 131 (1997).

[14] V. Giovannetti, L. Maccone, S. Lloyd and P. Shor eprint quant-ph/0307098

[15] J. Harrington and J. Preskill, Phys. Rev. A 64, 062301 (2001).

[16] S. Lloyd, Phys. Rev. A 55, 1613 (1997). 